\title{
Prediction of Performance for an Ejector Refrigeration Cycle Working with R245fa Using Artificial Neural Network
}

\author{
Mehdi Bencharif ${ }^{1, *}$, Sergio Croquer ${ }^{2}$, Sébastien Poncet ${ }^{2}$, Said Zid ${ }^{1}$, Hakim Nesreddine ${ }^{3}$ \\ ${ }^{1}$ Laboratoire du Génie Climatique Constantine, Université frères Mentouri, Constantine, Algeria \\ ${ }^{2}$ Mechanical Engineering Department, Université de Sherbrooke, Sherbrooke (QC), Canada \\ ${ }^{3}$ Laboratoire des Technologies de l'Énergie, Hydro-Québec, Shawinigan (QC), Canada \\ *Corresponding author : Mehdi.bencharif@umc.edu.dz
}

\begin{abstract}
In this paper, an artificial neural network (ANN) model is used to predict the performance parameters of an ejector refrigeration cycle working with R245fa. Three approaches are used to achieve this objective: experimental analysis, thermodynamic modeling, and artificial neural network. Fourteen parameters were collected from eight numerical or experimental studies. The ANN input parameters include geometric features $\left(\mathrm{D}_{\mathrm{col}}, \mathrm{D}_{\text {primout }}, \mathrm{NXP}, \mathrm{D}_{\mathrm{cas}}, \mathrm{L}_{\mathrm{cas}}, \mathrm{D}_{\text {out }}, \mathrm{L}_{\mathrm{diff}}\right)$ and operating conditions $\left(\mathrm{P}_{\text {prim }}, \mathrm{T}_{\mathrm{prim}}, \mathrm{P}_{\mathrm{sec}}, \mathrm{T}_{\mathrm{sec}}\right.$,

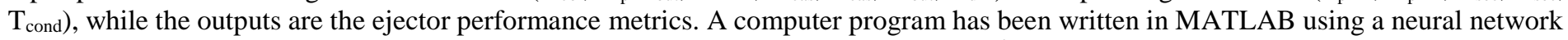
toolbox. The mean-square error (MSE) and the linear coefficient of correlation $\left(\mathrm{R}^{2}\right)$ have been chosen as metrics to evaluate the performance function and accuracy of the ANN model. In terms of the limiting compression ratio $\left(\mathrm{P}_{\text {cr }}\right)$ and entrainment ratio $(\omega)$, the ANN deviates by $3.63(\%)$ and $1.52(\%)$ respectively relative to the experimental data and by $-4.01(\%)$ and $-6.17(\%)$ relative to the thermodynamic model predictions.
\end{abstract}

Keywords: Artificial neural network, refrigeration cycle, ejector, R245fa.

\section{Introduction}

An ejector is a simple compression device with no moving parts. It is thermally activated by a high-temperature fluid stream, transferring energy to a low-pressure stream, propelling it in the process to intermediate conditions through the partial transfer of momentum. The resulting flow discharges at a stagnation pressure halfway between the primary and the secondary pressures [1]. A general schematics of a typical ejector geometry is shown in Figure 1. Ejectors are used in different fields (building, transportation, and industrial) due to their simplicity and reliability, requiring almost no maintenance and low operation costs [2]. In regards to refrigeration systems, they provide a promising alternative solution to conventional mechanical compression cycles, with lower energy consumption and reduced impact on the environment. Various investigations on ejectors have been conducted in the last decades. These, in general, assess the effects of operating conditions, different geometries, and working fluids on the performance of the device. Depending on the role of the device within the cycle, different configurations with varying performance and complexity have been proposed in the literature [1].

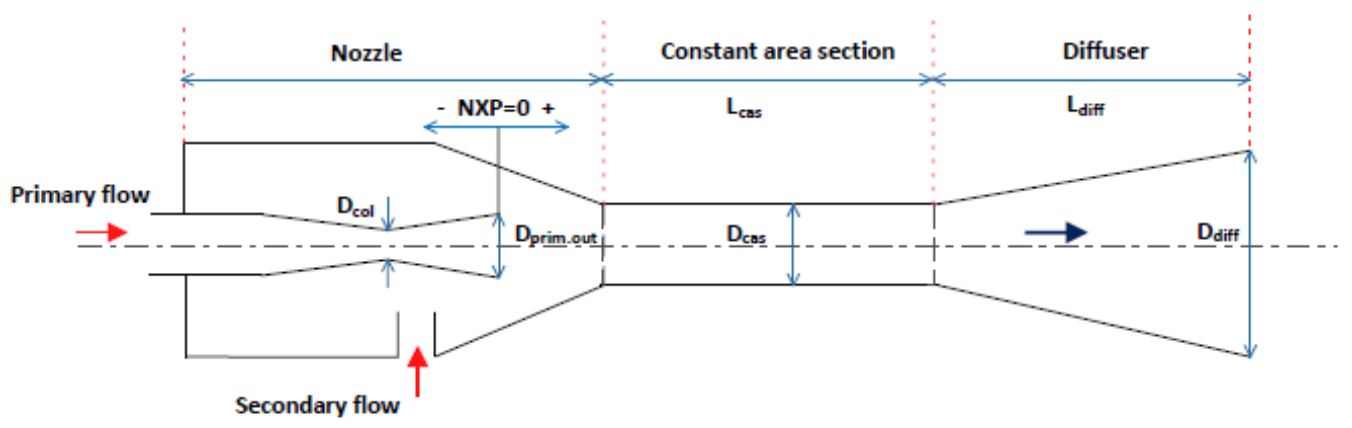

Fig. 1: Schematic view of the ejector geometry. 
There has been a great interest in recent years concerning the use of artificial neural networks in other fields [3][4]. It can be a solution for the design optimization of complex power cycles [5] and performance prediction and control design [6]. Boukelia et al. [7] present an optimization of a parabolic trough solar thermal power plant based on a feedforward back-propagation ANN model. Sozen and Arcaklioglu [8] have used ANN to calculate the exergy analysis of an ejector-absorption heat transformer; they have concluded that the ANN simplifies the thermodynamic analysis of the ejector-absorption heat transformer (EAHT) and reduces the need to solve systems of differential equations. Rashidi et al. [9] made a thermodynamic analysis of the ejector refrigeration cycle using an artificial neural network with R600a as a working fluid for predicting unknown operating points. This investigation aims to present an artificial neural network model to predict the performance of ejector refrigeration systems. The model uses 12 input parameters to predict the ejector entrainment and limiting compression ratios, respectively $(\omega)$ and $\left(\mathrm{P}_{\mathrm{cr}}\right)$. The different steps of training, testing, and validation of the model are carried out using an experimental database of ejector-based refrigeration cycles with R245fa as working fluid collected from literature reviews. In the following, the ANN architecture and the experimental data based are described. Then, the main results are presented and comparisons are carried between experimental, numerical, and ANN results to show the accuracy introduced ANN model.

\section{Artificial neural network}

Similar to the operation of a human brain, the ANN is constructed from cell-like structures, called perceptrons, which are linked together by weighted interactions. The architecture of the ANN is divided into three parts: input layers, hidden layers and output layers as shown in Figure 2. After random data division and before training, the data are normalized between -1 and +1 to make them consistent with the limits of a tangent sigmoid transfer function used in the hidden and output layers. The ANN models were trained, tested, and validated using back-propagation algorithm, in this network the Levenberg-Marquardt algorithm implemented in MATLAB R2014 software is used. To calibrate the weighting factors, a neural network needs to be trained by presenting it with a subset of the database and the corresponding output values. After that, test and validation sets are presented to the network at each cycle to select the best ANN. This is based on calculating the mean squared error (MSE), which tends to decrease as the number of training cycles or iterations increases, as given by the following equation:

$$
\mathrm{MSE}=\frac{1}{N} \sum_{i}\left|t_{i}-o_{i}^{2}\right|
$$

The mean squared error is the average squared difference between outputs $\left(\mathrm{t}_{\mathrm{i}}\right)$ and targets $\left(\mathrm{o}_{\mathrm{i}}\right)$. Lower values are better. Zero values mean no error solutions. $\mathrm{N}$ is the total number of data points. Once the objective error three should is achieved, the output results obtained for each model were compared to the corresponding actual results. The comparison consisted of calculating the coefficient of determination $\mathrm{R}^{2}$.

$$
R^{2}=1-\left(\frac{\sum_{i}\left(t_{i}-o_{i}\right)^{2}}{\sum_{i}\left(y_{i}\right)^{2}}\right)
$$




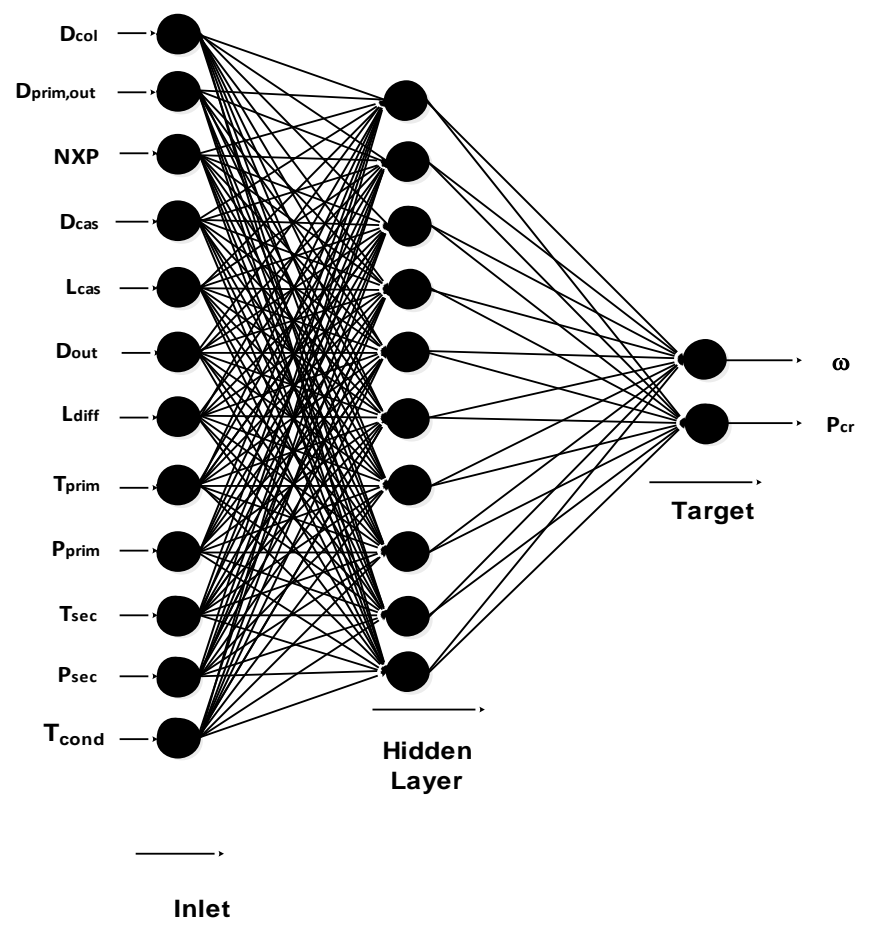

Fig. 2: Structure of the artificial neural network.

\section{Data base}

The database is built from a collection of 8 experimental studies on ejectors found in the literature [10-17]. This database includes 233 sets of data used to train, test, and validate the ANN model in order to predict the parametric performance (entrainment and compression ratio) of the ejector. Initially, twelve input parameters were selected. Namely, the pressure and the temperature of the primary and secondary streams $\left(\mathrm{P}_{\text {prim }}, \mathrm{T}_{\text {prim }}\right.$ and $\left.\mathrm{P}_{\text {sec }}, \mathrm{T}_{\mathrm{sec}}\right)$, the condenser temperature $\left(\mathrm{T}_{\text {cond }}\right)$, the primary nozzle throat diameter $\left(\mathrm{D}_{\mathrm{col}}\right)$, the primary nozzle outlet diameter $\left(D_{\text {prim,out }}\right)$, the nozzle exit position $(\mathrm{NXP})$, the constant area section diameter $\left(\mathrm{D}_{\text {cas }}\right)$, the constant area section length $\left(\mathrm{L}_{\text {cas }}\right)$ and the diffuser diameter $\left(\mathrm{D}_{\text {out }}\right)$ and length $\left(\mathrm{L}_{\text {diff }}\right)$. The values for all used parameters in the present ANN model are summarized in Table 1, with their minimum and maximum for each feature and the corresponding units.

Table 1: Input parameters with their minimum and maximum values.

\begin{tabular}{|c|c|c|c|}
\hline Parameters & Units & Min & Max \\
\hline $\mathrm{D}_{\text {col }}$ & $\mathrm{mm}$ & 2.20 & 20.20 \\
\hline $\mathrm{D}_{\text {prim,out }}$ & $\mathrm{mm}$ & 3.80 & 26.32 \\
\hline $\mathrm{NXP}$ & $\mathrm{mm}$ & 0.00 & 69.93 \\
\hline $\mathrm{D}_{\text {cas }}$ & $\mathrm{mm}$ & 12.16 & 34.07 \\
\hline $\mathrm{L}_{\text {cas }}$ & $\mathrm{mm}$ & 57.45 & 223.77 \\
\hline $\mathrm{D}_{\text {out }}$ & $\mathrm{mm}$ & 20.00 & 108.30 \\
\hline $\mathrm{L}_{\text {diff }}$ & $\mathrm{mm}$ & 46.00 & 950.00 \\
\hline $\mathrm{T}_{\text {prim }}$ & ${ }^{\circ} \mathrm{C}$ & 70.00 & 120.00 \\
\hline $\mathrm{P}_{\text {prim }}$ & $\mathrm{kPa}$ & 101.07 & 1921.00 \\
\hline $\mathrm{T}_{\text {sec }}$ & ${ }^{\circ} \mathrm{C}$ & 1.90 & 30.60 \\
\hline $\mathrm{P}_{\text {sec }}$ & $\mathrm{kPa}$ & 58.05 & 120.00 \\
\hline $\mathrm{T}_{\text {cond }}$ & ${ }^{\circ} \mathrm{C}$ & 14.88 & 63.80 \\
\hline
\end{tabular}




\section{Validation}

The training process greatly influences the success of the ANN models to obtain accurate output predictions. The performance of the ANN model is assessed via the convergence of MSE for training, validation, and testing. The evolution of this metric according to the number of iterations. The complete database was split into testing, validation, and training sets in a proportion of, respectively, 15\%, 15\%, and $70 \%$. The best validation performance was observed after 3 epochs (MSE 0.047), which confirms no overfitting observation. The plot of the linear coefficient of regression illustrated in Figure 3 indicates that all steps of training, testing and validation of the ANN model for the prediction of $(\omega)$ and $\left(\mathrm{P}_{\mathrm{cr}}\right)$ are obtained with successful results. The overall correlation coefficient $\left(\mathrm{R}^{2}\right)$ obtained in this investigation is 0.949 for training, 0.965 for testing and 0.978 for validation, which shows that the chosen ANN fits very well to the data, both during training and validation.
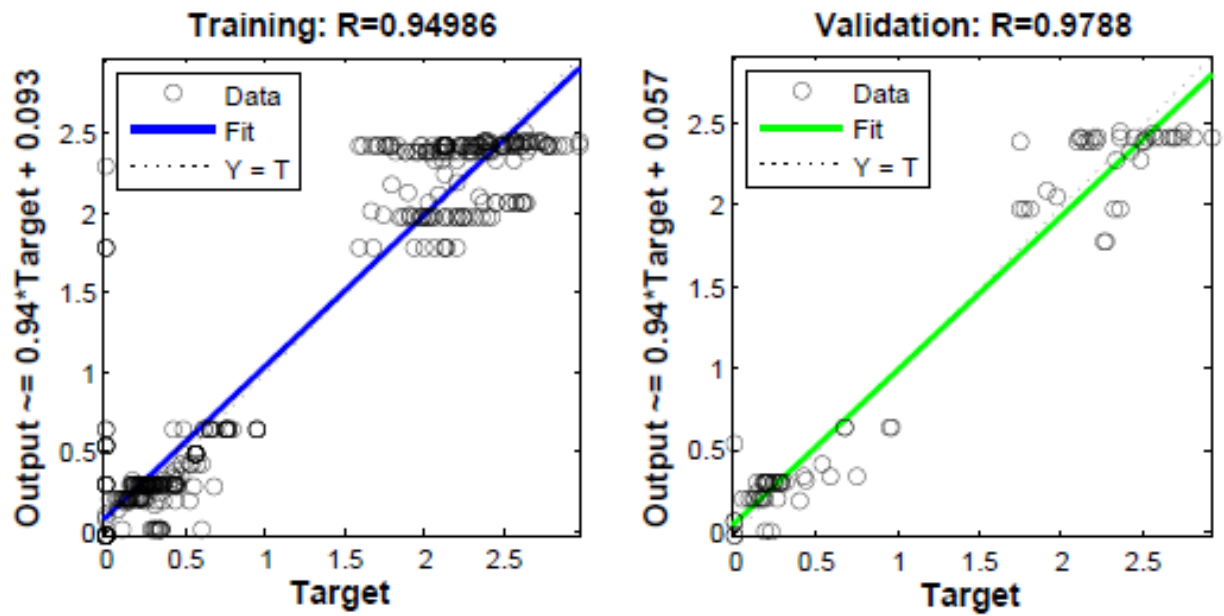

Test: $\mathbf{R}=0.96543$
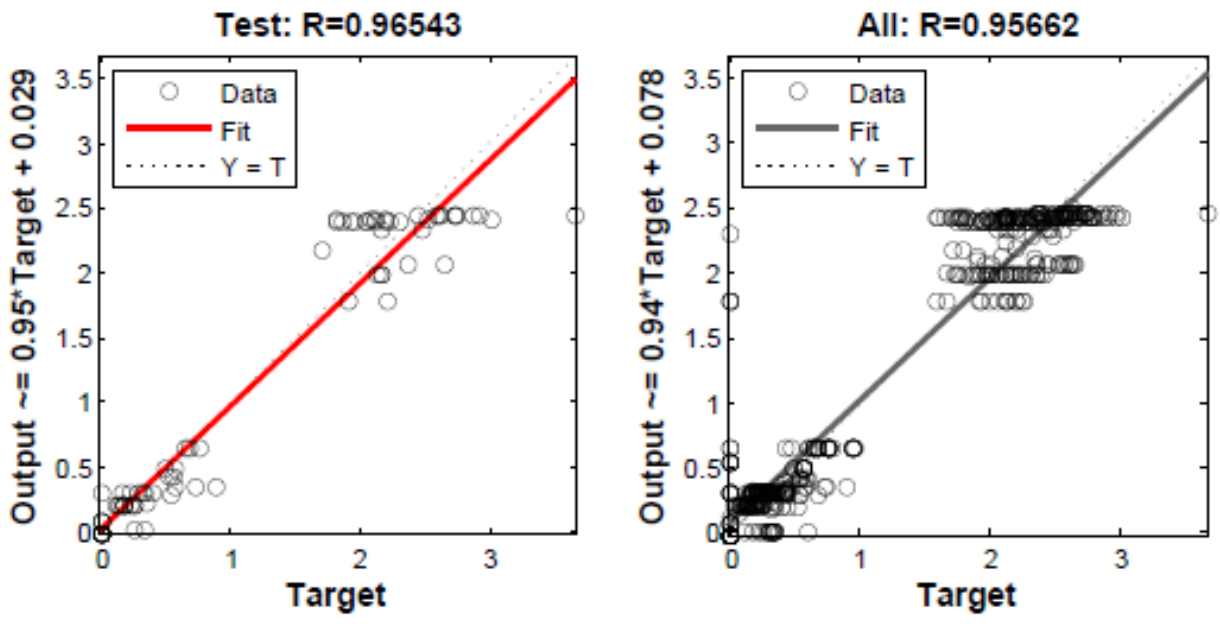

Fig. 3: Linear coefficient regression of the ANN model.

\section{Results and discussion}

The ANN model developed in this investigation to predict the performance parameters $(\omega)$ and (Pcr) shows promising results. To confirm the validation of the proposed working mechanism of the ANN, the predicted outputs are compared to experimental data extracted from $[11,15]$ and the predictions of a thermodynamic model detailed in $[11$, $15,18]$. From this data a new ANN model was made using 46 sets of data to make comparison between the three approaches: Experimental, Thermodynamic (Num) modelling and ANN as shown in Figure 4. Regarding the 
entrainment ratio (left side), the ANN predictions of $\omega$ follow the trends of the experimental data, although at some points it shows a greater deviation than the thermodynamic model and especially at lower condenser (ejector outlet) pressures. On the other hand, there is an overall fairly good agreement between the ANN results and the experimental data regarding the limiting compression ratio (right side).
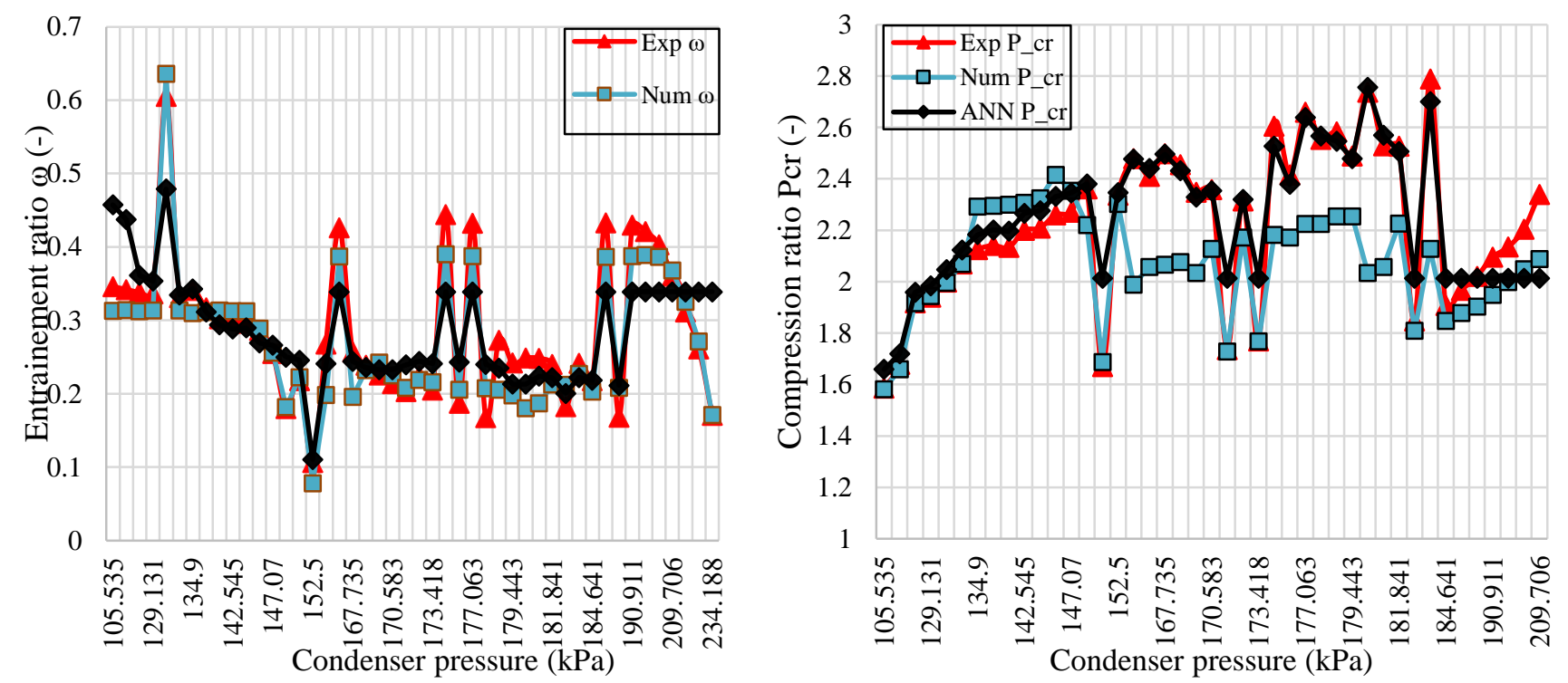

Fig. 4: Comparison between experimental $(\Delta)$, numerical $(\square)$ and $\operatorname{ANN}(\diamond)$ concerning $(\omega)$ in left and $\left(\mathrm{P}_{\text {cr }}\right)$ on right.

\section{Conclusion}

This paper presents a new ANN model to predict the performance of an ejector working with R245fa. Comparisons are carried out versus experimental and thermodynamic modelling results to validate the predicted ANN. The main conclusions can be summarized as follows:

- The ANN model proposed shows a strong learning ability and a good generalization performance.

- Compared with the experimental data, the ANN shows very good agreement (relative error of, respectively, 3.63

(\%) and $1.52(\%)$ for $\omega$ and Pcr), which outperforms the thermodynamic model approach.

\section{Acknowledgements}

This project is a part of the collaboration between the Université de Sherbrooke (Canada) and the Laboratoire de Génie Climatique de Constantine (LGCC) at Université des frères Mentouri in Constantine (Algeria). The authors would like to thank the Hydro-Québec research laboratory for the support provided during the experiments. S.P. acknowledges the NSERC chair on industrial energy efficiency established at Université de Sherbrooke with the support of Hydro-Québec, Ressources Naturelles Canada and Emerson Canada.

\section{References}

[1] Z. Aidoun, K. Ameur, M. Falsafioon, and M. Badache, "Current Advances in Ejector Modeling, Experimentation and Applications for Refrigeration and Heat Pumps. Part 1: Single-Phase Ejectors," Inventions, vol. 4, no. 1, 2019.

[2] G. Singhal, A. Dawar, and P. Subbarao, "Application of profiled ejector in chemical lasers," Appl. Therm. Eng., vol. 28, no. 11-12, pp. 1333-1341, 2008.

[3] N. D. Roman, F. Bre, V. D. Fachinotti, and R. Lamberts, "Application and characterization of metamodels based on artificial neural networks for building performance simulation: a systematic review," Energy \& Buildings, vol. 217, 109972, 2020. 
[4] A. R. Pazikadin, D. Rifai, K. Ali, M. Z. Malik, A. N. Abdalla, and M. Faraj, "Solar irradiance measurement instrumentation and power solar generation forecasting based on Artificial Neural Networks (ANN): A review of five years research trend," Science of The Total Environment. vol. 715, pp. 136848, 2020.

[5] O. Arslan and O. Yetik, "ANN based optimization of supercritical ORC-Binary geothermal power plant: Simav case study," Appl. Therm. Eng., vol. 31, no. 17-18, pp. 3922-3928, 2011.

[6] A. Afram, and F. Janabi-Sharifi, "Black-box modeling of residential HVAC system and comparison of gray-box and black-box modeling methods," Energy \& Buildings, vol. 94, pp. 121-149, 2015.

[7] T. Boukelia, O. Arslan, and M. Mecibah, "Potential assessment of a parabolic trough solar thermal power plant considering hourly analysis: ANN-based approach," Renewable Energy, vol. 105, pp. 324-333, 2017.

[8] A. Sözen and E. Arcaklioğlu, "Exergy analysis of an ejector-absorption heat transformer using artificial neural network approach," Appl. Therm. Eng., vol. 27, no. 2-3, pp. 481-491, 2007.

[9] M. Rafiq, G. Bugmann, and D. J. Easterbrook, "Neural network design for engineering applications," Comput. \& Structures, vol. 79, no. 17, pp. 1541-1552, 2001.

[10] M. Hamzaoui, H. Nesreddine, Z. Aidoun, and M. Balistrou, "Experimental study of a low grade heat driven ejector cooling system using the working fluid R245fa," Int. J. Refrig., vol. 86, pp. 388-400, 2018.

[11] P. Haghparast, M. V. Sorin and H. Nesreddine, "Effects of component polytropic efficiencies on the dimensions of monophasic ejectors," Energy Convers. Manag., vol. 162, pp. 251-263, 2018.

[12] P. Haghparast, M. V. Sorin, and H. Nesreddine, "The impact of internal ejector working characteristics and geometry on the performance of a refrigeration cycle," Energy, vol. 162, pp. 728-743, 2018.

[13] K. Shestopalov, B. Huang, V. Petrenko, and O. Volovyk, "Investigation of an experimental ejector refrigeration machine operating with refrigerant R245fa at design and off-design working conditions. Part 2. Theoretical and experimental results," Int. J. Refrig., vol. 55, pp. 212-223, 2015.

[14] F. Mazzelli and A. Milazzo, "Performance analysis of a supersonic ejector cycle working with R245fa," Int. J. Refrig., vol. 49, pp. 79-92, 2015.

[15] D. Scott, Z. Aidoun, and M. Ouzzane, "An experimental investigation of an ejector for validating numerical simulations," Int. J. Refrig., vol. 34, no. 7, pp. 1717-1723, 2011.

[16] I. W. Eames, A. E. Ablwaifa, and V. Petrenko, "Results of an experimental study of an advanced jet-pump refrigerator operating with R245fa," Appl. Therm. Eng., vol. 27, no. 17-18, pp. 2833-2840, 2007.

[17] I. W. Eames, A. Milazzo, D. Paganini, and M. Livi, "The design, manufacture and testing of a jet-pump chiller for air conditioning and industrial application," Appl. Therm. Eng., vol. 58, no. 1-2, pp. 234-240, 2013.

[18] M. Bencharif, H. Nesreddine, S. Croquer, S. Poncet, and S. Zid, "The benefit of droplet injection on the performance of an ejector refrigeration cycle working with R245fa," Int. J. Refrig., vol. 113, pp. 276-287, 2020. 